\title{
One target for amyotrophic lateral sclerosis therapy?
}

Targeting a single protein reduces both toxic repeat RNAs and proteins

By Sarah Mizielinska and Adrian M Isaacs

Department of Neurodegenerative Disease, UCL Institute of Neurology, London WC1N 3BG, UK. Email: a.isaacs@.ucl.ac.uk

Repeat expansion mutations cause a range of developmental, neurodegenerative, and neuromuscular disorders. The repeat sequences generally comprise a 3- to 6-base pair repeat unit that expands above a critical threshold, leading to disease. Expanded repeats cause disease via a range of mechanisms, including loss of function of the repeat-containing protein and production of toxic repeat RNAs and proteins, making the disorders difficult to treat. In 2011, a hexanucleotide repeat expansion in the C9orf72 gene was identified as the most common cause of frontotemporal dementia and amyotrophic lateral sclerosis (termed C9FTD/ALS) $(1,2)$. On page XXX of this issue, Kramer et al. (3) report that targeting a single factor, Spt4, reduced production of C9orf 72 repeat expansion-associated RNA and protein, and ameliorated neurodegeneration in model systems.

Kramer et al.'s use of a single factor to reduce multiple repeat-associated pathologies is notable in the light of two unexpected features of repeat expansions. One is that repeat expansions are transcribed in both the antisense and sense direction (X). The other is that repeat-associated nonATG (RAN) translation occurs, in which repeat expansions mediate their own translation into proteins (4). As no ATG start codon is required, RAN translation can occur in all six sense and antisense frames. A major therapeutic challenge is to target the wide range of potentially toxic RNA and protein species that are produced.

The yeast Spt4 (human homolog SUPT4H1) is a small, evolutionarily conserved zinc finger protein that forms a complex with Spt5. The Spt4-Spt5 complex binds to RNA polymerase II and regulates transcriptional elongation. Deletion of Spt4 in yeast was shown to reduce the transcription of expanded CAG, CTG, and CAA repeats, but had little effect on short repeats (5). Similar effects were observed with CAG repeats (which cause Huntington's disease) in cultured mouse neurons. In addition, depletion of Supt4h in two different mouse models of Huntington's disease (6) selectively reduced transcription of the repeat expansion allele while leaving transcription of the normal allele unaffected. This decreased mutant, but not wild-type, protein production and aggregation, delayed onset of motor phenotypes, and prolonged life span.

Kramer et al. now extend this work to C9orf72 GGGGCC repeat expansions. In C9FTD/ALS, both sense and antisense repeat RNA transcripts form aggregates, termed RNA foci, in patient brains. RNA foci exert toxicity in other repeat expansion diseases by sequestering essential RNA-binding proteins (7). Using yeast models expressing either expanded sense or antisense C9orf72 repeats, Kramer et al. found that Spt4 depletion decreased the number of both sense and antisense repeat transcripts and RNA foci.

C9orf72 RAN translation leads to the production of five dipeptide repeat proteins that can cause neurodegeneration in model systems (8). Kramer et al. showed that production of one of them, poly(glycine-proline), was substantially reduced by Spt4 depletion in yeast and worm C9orf72 models, as would be expected if less repeat RNA was available for translation. Reducing the 
expression of Spt4 also improved survival in C9orf72 worm and fruit fly models, indicating a reduction of toxic repeat species.

To study endogenous expanded repeats, Kramer et al. used human C9ALS patient fibroblasts. Reducing the expression of either human SUPT4H1 or its binding partner SUPT5H (homolog of Spt5), or both, reduced C9orf72 sense and antisense RNA foci and poly(glycine-proline) levels. Additionally, reducing SUPT4H1 expression in C9ALS patient-induced pluripotent stem cell-derived cortical neurons reduced the amount of C9orf72 transcripts and poly(glycine-proline). Thus, targeting human SUPT4H1 and SUPT5H can effectively reduce multiple key C9FTD/ALS pathologies (see the figure).

One concern for SUPT4H1 as a therapeutic target is that it may regulate other non-mutated genes. Deletion of Spt4 in yeast changed the regulation of 149 genes com-pared to controls (4). In the study of Kramer et al., 95\% depletion of SUPT4H1 in human fibroblasts altered the expression of 301 genes. Of note, deletion of one copy of Supt4h did not exhibit any overt phenotype in mice up to 18 months of age, but deletion of both copies is embryonic lethal (5). Thus, the degree of SUPT4H1 depletion will be critical for effective therapy development.

One of the most advanced potential therapeutics for repeat expansion disorders, including Huntington's disease and C9FTD/ALS, are antisense oligonucleotides that specifically target the mutant expanded allele. Compared to mutant gene-specific antisense oligonucleotides, potential advantages of the SUPT4H1-targeting strategy are its wider applicability and the reduction of both sense and antisense transcripts. However, the relative role of antisense RNA and protein species in disease pathogenesis is currently unclear, so targeting sense repeats may still have a beneficial effect. In this context, Kramer et al. show that an antisense oligo-nucleotide targeting the sense C9orf72 strand almost completely reduced poly(glycine-proline) concentrations levels in patient fibroblasts. In addition, antisense oligonucleotides that specifically target the gene of interest may have fewer off-target effects. As the authors suggest, an exciting possibility is the development of an anti-sense oligonucleotide targeting SUPT4H1, particularly because this may have broad potential for repeat expansion diseases.

\section{REFERENCES}

1. M. Dejesus-Hernandez et al., Neuron 72, 245 (2011).

2. A. E. Renton et al., Neuron 72, 257 (2011).

X. R. Batra et al., Hum. Mol. Genet. 19, (2010).

3. N. J. Kramer et al., Science 353, XXX (2016).

4. Zu et al., Proc. Natl. Acad. Sci. U.S.A. 108, 260-5 (2011).

5. C. R. Liu et al., Cell 148, 690- (2012).

6. H.-M. Cheng et al., PLOS Genet. 11, e1005043 (2015).

7. M. Wojciechowska et al., Hum. Mol. Genet. 20, 3811-21 (2011).

8. Mizielinska et al., Science 345, 1192-1194 (2014). 


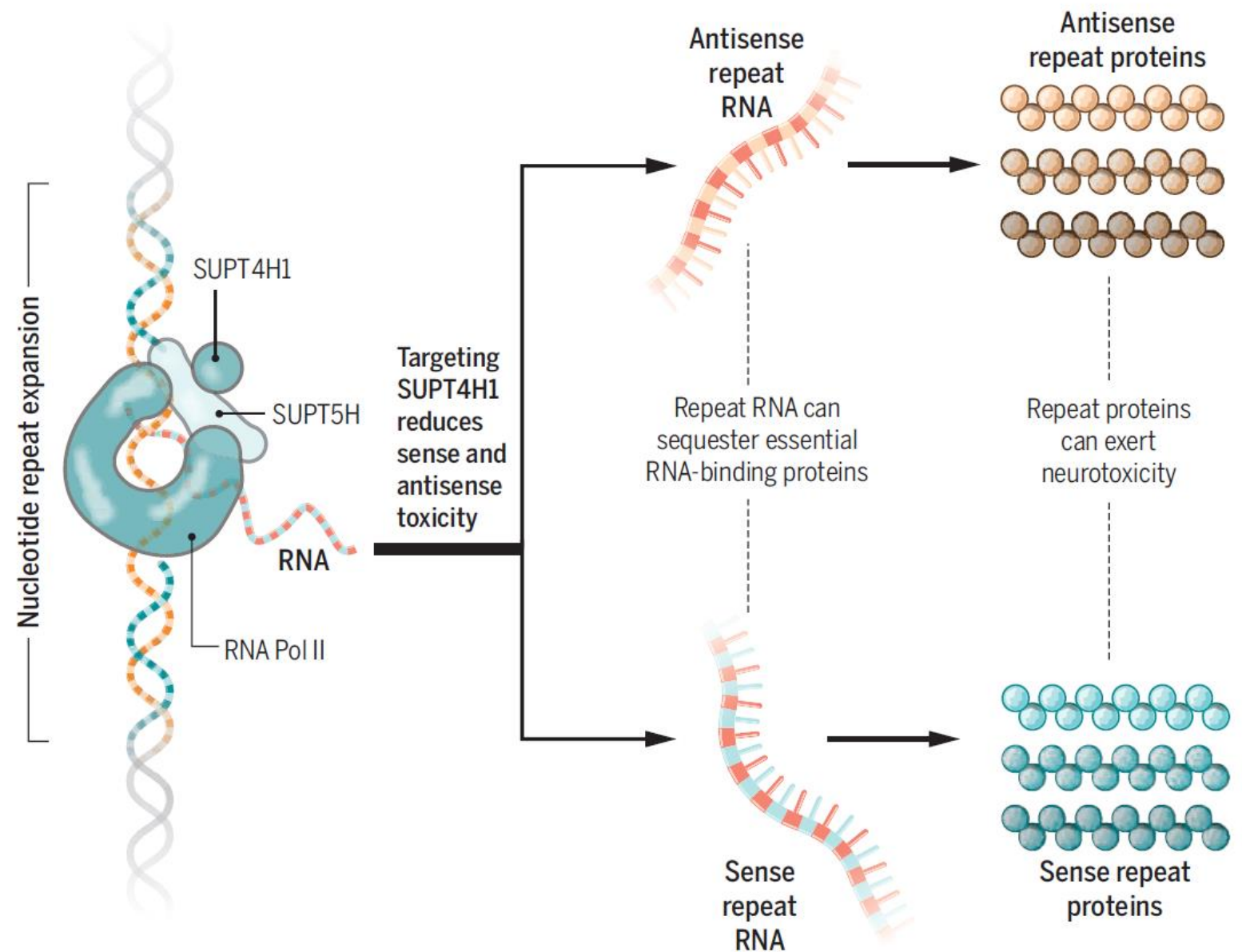

Root cause. The SUPT4H1-SUPT5H complex binds RNA polymerase II and regulates transcription elongation of expanded nucleotide repeats, which cause a range of diseases including ALS. Targeting SUPT4H1 reduces production of multiple toxic species, specifically sense and antisense repeat RNAs and repeat proteins. 